\title{
Gulf Stream Dynamics: A Note
}

\section{Kern E. Kenyon}

4632 North Lane, Del Mar, CA, USA

Correspondence to: Kern E. Kenyon, kernken@aol.com

Keywords: Bernoulli's Law, Gulf Stream

Received: April 29, $2019 \quad$ Accepted: May 24, 2019

Published: May 27, 2019

Copyright (c) 2019 by author and Scientific Research Publishing Inc.

This work is licensed under the Creative Commons Attribution International License (CC BY 4.0).

http://creativecommons.org/licenses/by/4.0/

\section{Open Access}

\section{ABSTRACT}

\begin{abstract}
A preliminary attempt is made to address Stommel's suggestion that the established offshore shear in the Gulf Stream's surface velocity is large (up to $0.5 f$, where $f$ is the Coriolis parameter) and that this needs to be understood better. Using Bernoulli's law in conjunction with the geostrophic relation leads to the prediction that the shear should equal $f$. If a certain sea level variation across the Stream is proposed within the warm Stream water, it is qualitatively possible to reduce the shear value below $f$, but more work in the future will confirm or refute this idea.
\end{abstract}

\section{INTRODUCTION}

Nowhere until now has the Gulf Stream and Bernoulli's law occurred in the same sentence, to the best of my knowledge. In a steady fluid motion, where the speed is greatest, the pressure is least, and vice versa, along streamlines, it has not so far found a place there.

What stimulates initiating the present subject is a recent tentative notion that Bernoulli's law may actually be involved in certain weather events [1]. And that association happened by accident. Across the North Pacific along $35 \mathrm{~N}$, an oceanographic ship sailed west in the spring of 1976. Though dedicated to the physics and chemistry of the entire water column, routine meteorological measurements were also made on the bridge every two hours around the clock for 35 consecutive days.

Within all weather data recorded quantitatively, there was unexpectedly revealed a prominent variation with a time-scale of two days [2]. Then years later, it was noticed that the air pressure and wind speed records were 180 degrees out of phase at the two day cycle, which led to the idea that Bernoulli's law may have been operating during that time [3].

Separate studies of flow past a cylinder or a sphere [4] have shown that Bernoulli's law along streamlines can be combined with another equation for the balance of forces across streamlines: centrifugal force on the curved paths equals a pressure gradient. Then, the two equations in two unknowns (pressure and flow speed) can be solved by elimination of one of the variables to get one equation in one unknown.

When the horizontal scale of the flow phenomenon increases past the point where, in the force balance equation, the centrifugal force should be replaced by the Coriolis force, and no other forces are add- 
ed, then elimination of the pressure between it and Bernoulli's equation produces a very simple relation: horizontal velocity shear equals the Coriolis parameter [4].

Quoting from Stommel's Gulf Stream book (first edition): "The general fact is that a wide zone of anticyclonic vorticity (of approximately $-0.5 f$, where $f$ is the local Coriolis parameter) seems to be established and also requires explanation." (A translation of vorticity there [5] is velocity shear here.) An understanding for an even larger current shear, exactly equal to $f$, is given below, along with discussions of how it could be made smaller by adding a force to one of the two governing equations that accounts for a particular sea level variation.

\section{METHOD}

Start with Bernoulli's law

$$
p=\text { const }-\frac{1}{2} \rho V^{2}
$$

where $p$ is the pressure, $V$ is the flow speed, $\rho$ is the fluid density and the same constant is assumed to apply to all streamlines for simplicity.

Geostrophy is written

$$
-\frac{\mathrm{d} p}{\mathrm{~d} x}=\rho f V
$$

where the pressure force is on the LHS and the Coriolis force is on the RHS, $f$ being the Coriolis parameter. Assume the flow is into the page, $x$ is positive to the right and the pressure decreases to the left.

Equations (1) and (2) are two equations in the two unknowns $p, V$. Take the $\mathrm{x}$-derivative of (1) and use that to eliminate the pressure in (2) resulting in

$$
\frac{\mathrm{d} V}{\mathrm{~d} x}=f
$$

which in condensed form says that the horizontal velocity shear equals the Coriolis parameter.

\section{DISCUSSION}

Equation (3) has recently been applied to a certain weather event in the North Pacific and found to be consistent with observations from a wind storm [1], but it is not yet ready to be applied to the Gulf Stream. The reason is that (3) is not complete enough for that job: there is at least one term missing from (1) and/or a force absent from (2). Not only that, but the exact form of the missing information is not known well enough to be expressed algebraically at this time.

Since the Gulf Stream imports warmer water from lower latitudes into a lower temperature environment, a guess can be made that the sea level within the Gulf Stream is higher than it is outside the current, caused by a density variation in its water column. There may exist sea level data to show whether or not this guess is correct, but if so I have not seen them.

Suppose a sea level variation actually occurs within the Gulf Stream region, in the cross-stream direction. Then a force on the surface water will exist in that direction and point down slope. For a given shoreward pressure force on the LHS of (2), adding an offshore force due a sea a level variation on the RHS means the Coriolis force must be smaller to maintain the balance. In other words, effectively the Coriolis parameter is smaller or the shear is smaller than $f$.

How could the sea level be highest in the middle of the Gulf Stream with an offshore downward slope on the right side? Here is a proposed density arrangement that could work. Perhaps the fastest flow brings into the region the warmest surface water because there has been less time in transit for heat to leak out into the air. Assume the water column containing the highest SSTs is short compared to the water column to the right in mid-stream. Therefore, the larger water column, although having a slightly lower temperature will produce a higher sea level because of its greater thickness. Essentially the lighter density warm 
Gulf Stream water is floating on the colder environmental water underneath, and it is held up to the surface by the buoyancy force associated with the thermocline. Sea level will be highest in the middle of the Stream. These qualitative comments need to be made quantitative as soon as possible.

\section{CONCLUSION}

Bernoulli's law is brought to the Gulf Stream, along with the geostrophic relation, in order to try to increase the understanding of the large surface current shear on the offshore side of the Stream. In the future, other factors may need to be added, because actually Bernoulli provides too much help. One feature that has the potential to reduce the strength of Bernoulli's law is a higher sea level inside the Stream due to the lower density in the warm water column.

\section{CONFLICTS OF INTEREST}

The author declares no conflicts of interest regarding the publication of this paper.

\section{REFERENCES}

1. Kenyon, K.E. (2019) A Bernoulli-Geostrophy Weather Event. Natural Science, 11, 122-125. https://doi.org/10.4236/ns.2019.114013

2. Kenyon, K.E. (1996) Bi-Daily Variation of Meteorological Properties at Sea Level across the Pacific along 35 N. Atmospheric Research, 43, 31-56. https://doi.org/10.1016/S0169-8095(96)00003-8

3. Kenton, K.E. (2018) Bernoulli Weather or Not? Natural Science, 10, 178-181. https://doi.org/10.4236/ns.2018.105019

4. Kenyon, K.E. (2019) Flow Past a Sphere. Natural Science, 11, 95-97. https://doi.org/10.4236/ns.2019.113010

5. Stommel, H. (1960) The Gulf Stream. University of California Press, Los Angeles, p. 139. 